\title{
Postoperative pain control after spinal surgery: an opportunity
}

\author{
Zoher Ghogawala, MD \\ Department of Neurosurgery, Lahey Hospital and Medical Center, Tufts University School of Medicine, Burlington, \\ Massachusetts
}

$\mathrm{T}$ HE importance of achieving pain control after spinal surgery has been emphasized by many. Adequate postoperative pain control is associated with improved patient satisfaction, reduced postoperative opioid dependence, shorter hospital length of stay, and lower costs. ${ }^{1}$ In the current issue of the Journal of Neurosurgery: Spine, Yang et al. use data from the Canadian Spine Outcomes and Research Network (CSORN) to develop a predictive model for identifying patients who had a high or extremely high risk of having inadequate pain control after spinal surgery. ${ }^{2}$ In their study, $57 \%$ of patients experienced inadequate pain control in the first 24 hours after surgery.

The authors used advanced statistical techniques to evaluate 910 patients to develop their model. Using 25 clinical variables, they identified 7 significant ones that were used to develop a simplified 3-tier score to predict risk. In the low-risk tier, patients had a $32 \%$ risk of having inadequate pain control (defined as a mean numeric rating scale [NRS] score for pain of $>4$ during the first 24 hours after surgery). In the high-risk tier, $63 \%$ of patients had inadequate pain control, and for patients in the extremely high-risk category, $85 \%$ of patients had inadequate pain control.

The investigators validated their model with $30 \%$ of the data (390 patients). Similar results were obtained. Nonmodifiable risk factors included younger age and female sex. Other identified risk factors may or may not be modifiable: preoperative opioid usage (32\% of the population), higher Patient Health Questionnaire-9 depression score, fusion surgery, surgery involving 3 or more motion segments, and higher preoperative pain scores.

In many cases, we have choices around which surgical procedure is optimal for specific patients. The threshold for recommending a fusion and for surgically treating 3 or more motion segments differs widely among spinal surgeons. Involving patients and including pain control in the discussion might help educate patients about the benefits and alternatives around the comparative advantages and disadvantages of specific surgical decisions.

The authors suggest that preoperative opioid deprescribing programs and psychotherapy methods, including acceptance and commitment therapy, might help to reduce inadequate postoperative pain control. ${ }^{3,4}$ Clearly, further study is needed to determine the effectiveness of attempting to modify some risk factors that have a significant behavioral component.

The authors have done a nice job in highlighting the importance of studying postoperative pain control after spinal surgery. There is no question that focusing on patient-centric aspects of care will improve our ability to generate improved outcomes. This study raises many important issues that deserve further study. The assessment of pain control was not standardized in this study. Patients had an average of 9.2 assessments during the first 24 hours. Patients who had NRS scores $>4$ but had very satisfactory responses to analgesia might be very different from patients who had suboptimal responses to postoperative analgesia. Determining how best to assess this will require a careful approach. The impact of suboptimal pain control in the first 24 hours and its possible effect on hospital length of stay and on the utilization of postoperative opioids may be more complicated than it might appear. Developing protocols to optimize pain control may not change some of the underlying factors driving longer length of stay and opioid dependence after surgery.

It is essential to have further studies that focus on strategies to improve postoperative pain control in the future. This study by Yang et al. provides some direction by identifying potentially modifiable risk factors. The next step 
will be to determine how to manage this information and if modification is feasible and effective in optimizing postoperative pain control.

https://thejns.org/doi/abs/10.3171/2020.6.SPINE20803

\section{References}

1. Gerbershagen HJ, Pogatzki-Zahn E, Aduckathil S, et al. Procedure-specific risk factor analysis for the development of severe postoperative pain. Anesthesiology. 2014;120(5): 1237-1245.

2. Yang MMH, Riva-Cambrin J, Cunningham J, et al. Development and validation of a clinical prediction score for poor postoperative pain control following elective spine surgery. $J$ Neurosurg Spine. Published online September 15, 2020. doi: 10.3171/2020.5.SPINE20347

3. Nguyen L-CL, Sing DC, Bozic KJ. Preoperative reduction of opioid use before total joint arthroplasty. J Arthroplasty. 2016;31(9)(suppl):282-287.

4. Abid Azam M, Weinrib AZ, Montbriand J, et al. Acceptance and Commitment Therapy to manage pain and opioid use after major surgery: preliminary outcomes from the Toronto General Hospital Transitional Pain Service. Can J Pain. 2017;1(1):37-49.

\section{Disclosures}

The author reports no conflict of interest.

\section{Correspondence}

Zoher Ghogawala: zoher.ghogawala@lahey.org.

INCLUDE WHEN CITING

Published online September 15, 2020; DOI: 10.3171/2020.6.SPINE20803.

\section{Response}

\section{Michael M. H. Yang, MD, MSc, MBiotech, ${ }^{1}$ Jay Riva-Cambrin, MD, MSc, ${ }^{1}$ and Steven Casha, MD, PhD ${ }^{1,2}$}

${ }^{1}$ Department of Clinical Neurosciences, Section of Neurosurgery, and ${ }^{2}$ Hotchkiss Brain Institute, University of Calgary, Alberta, Canada

Our study identified 7 preoperative and surgical predictors of poor postoperative pain control after elective spine surgery. Five predictors (younger age, female sex, preoperative pain, depression, and opioid use) have also been associated with a poor postoperative pain experience across a variety of surgical disciplines. ${ }^{1}$ Two variables (surgery at $\geq 3$ motion segments and spinal fusion), are specific risk factors in the spine surgery population.

As Dr. Ghogawala has suggested, the resultant Calgary Postoperative Pain After Spine Surgery (CAPPS) score may be useful in educating patients about their expected short-term postoperative pain experience. While that discussion may influence the decision around surgical options, the relationship between the postoperative pain experience and long-term outcomes are less clear. Furthermore, we agree that the interplay between postoperative pain and other adverse outcomes, including opioid dependency and lengthy hospital stay, is complex and confounded by a multitude of other risk factors. Ultimately, the CAPPS score's impact on future patients' short-term pain outcomes will depend on our ability to substantially improve modifiable variables therein, such as depression and preoperative opioid use.

Clearly, optimizing patients' pain experience is an important objective. In time, validated Enhanced Recovery After Surgery protocols and personalized preoperative and perioperative treatment strategies to improve pain will result from our improved understanding of the patient experience. The CAPPS score may assist investigators studying such strategies in better controlling the large variability in the pain experience. However, Dr. Ghogawala has highlighted two further obstacles to these studies. First, the assessment of pain is oversimplified, inherently subjective, inconstant, and easily biased. Second, it is difficult to control for the influence of analgesia on the reported outcome due to variability in the prescription and in the patients' responsiveness. These are both future studies in our line of inquiry to optimize spine surgery patients' postoperative pain experience.

\section{References}

1. Yang MMH, Hartley RL, Leung AA, et al. Preoperative predictors of poor acute postoperative pain control: a systematic review and meta-analysis. BMJ Open. 2019;9(4):e025091.

\section{INCLUDE WHEN CITING}

Published online September 15, 2020; DOI: 10.3171/2020.6.SPINE201006. 